\title{
Integrando la competencia transversal responsabilidad ética, medioambiental y profesional en una asignatura de Máster
}

\author{
María de Miguel Molina ${ }^{a}$, Blanca de Miguel Molina ${ }^{b}$, Ángel Peiró Signes ${ }^{c}$ y Marival \\ Segarra Oña ${ }^{d}$ \\ ${ }^{a}$ Departamento de Organización de Empresas, Universitat Politècnica de València, \\ mademi@omp.upv.es, 'bepartamento de Organización de Empresas, Universitat Politècnica de \\ València, bdemigu@omp.upv.es, ' ${ }^{\circ}$ Departamento de Organización de Empresas, Universitat \\ Politècnica de València, anpeisig@omp.upv.es, ${ }^{\mathrm{d}}$ Departamento de Organización de Empresas, \\ Universitat Politècnica de València, maseo@omp.upv.es
}

\begin{abstract}
Usually, it is difficult to include in Master level some activities to promote the general competence "professional and social responsibility", because at the academic environment we frequently prepare activities to apply technical knowledge rather than "common sense". However, experiential learning, involving the student in a "real case study", can help them to interiorize that ethical knowledge in a practical way. Moreover, using learning tools such as LEGO Serious Play ${ }^{\circledR}$ enhances participation and decision-making in groups. Our goal is that the master students will be able to make a decision that affects people's lives in the most human way, without losing the opportunity that some technical advances can represent to improve the welfare of everybody.
\end{abstract}

Keywords: ethical responsibility, environmental responsibility, professional responsibility, experiential learning, sustainable development goals

\section{Resumen}

El integrar en las asignaturas de máster actividades que promuevan la competencia transversal "responsabilidad profesional y social" no es una tarea sencilla. En el ámbito académico, no siempre se dan situaciones en las que el alumno deba aplicar su "sentido común" sino más bien conocimientos técnicos. Sin embargo, el aprendizaje experiencial en el que el alumno se vea envuelto en un caso "real", puede servir para que interiorice estos conocimientos de una manera práctica. Además, el uso de metodologías como LEGO Serious Play ${ }^{\circledR}$ fomenta la participación y la toma de decisiones en grupo. Nuestro objetivo es que los alumnos de máster sean capaces de tomar una decisión que afecta a las personas de la manera más humana posible, 
sin perder de vista la oportunidad que determinados avances tecnológicos pueden prestar para mejorar la calidad de vida de todas las personas.

Palabras clave: responsabilidad ética, responsabilidad medioambiental, responsabilidad profesional, aprendizaje experiencial, objetivos de desarrollo sostenible.

\section{Introducción.}

Las competencias genéricas o transversales, representan una combinación dinámica de conocimiento, comprensión, habilidades y aptitudes (European Union, 2017). Dentro de ellas, la competencia "responsabilidad ética, medioambiental y profesional", se refiere al conjunto de conocimientos, habilidades, destrezas y actitudes (Universitat Politècnica de València, 2017):

- Útiles para interactuar con el entorno, en orden a evitar o disminuir los efectos negativos producidos por las prácticas inadecuadas que ocasiona la actividad humana y para promover los beneficios que pueda generar la actividad profesional en el ámbito medioambiental, teniendo en cuenta sus implicaciones económicas y sociales.

- Que orientan la acción humana en un sentido racional, por lo que se relaciona con las acciones y su valor moral.

- Que posibilitan una valoración positiva o negativa por el impacto ecológico de una decisión o no decisión (generalmente, al daño causado a otras especies, a la naturaleza o a las futuras generaciones), sea individual o en grupo.

- Que nos ayudan a interiorizar en nuestra conciencia una responsabilidad con dos objetivos primordiales: evitar toda falta voluntaria y disminuir, en lo posible, el número de faltas involuntarias por debilidad humana, flaqueza propia o negligencia ajena.

En este caso particular, para los alumnos del Máster Universitario en Gestión de Empresas, Productos y Servicios de la Facultad de ADE, hemos seguido un esquema circular, en el que la ética es un concepto que se aplica tanto de manera individual como colectiva, lo que hace que el beneficio sea mutuo. Si el individuo en su vida normal, como persona y ciudadano, actúa de manera ética, lo hará también a nivel profesional. Despúes, llevará esa práctica a la empresa y la empresa beneficiará a la sociedad. De este modo, esa cultura social se irá transmitiendo a los individuos que, en ese contexto, sabrán qué actuaciones son éticas y cuáles no. Así, el ciclo se retroalimenta (Figura 1).

(cc) EY-NC-ND 2018, Universitat Politècnica de València

Congreso IN-RED (2018) 


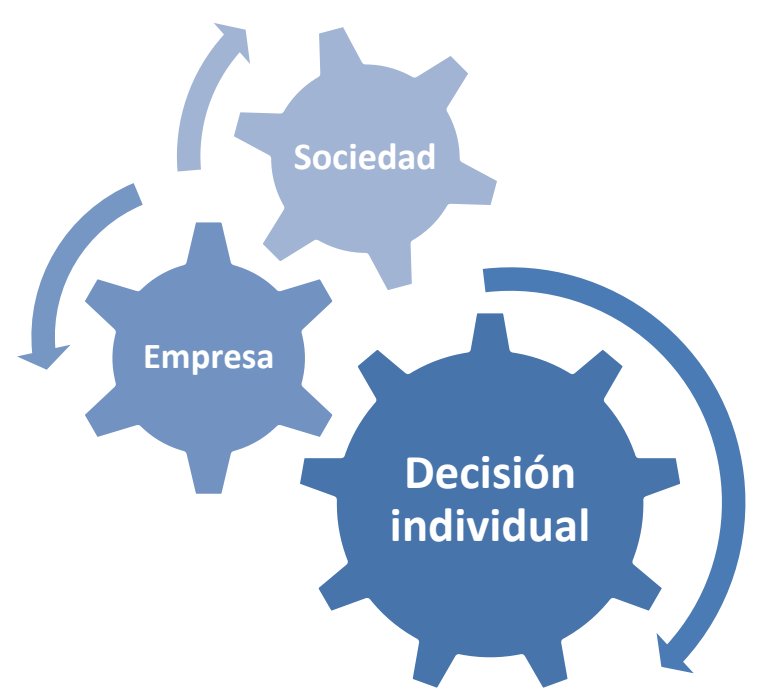

Fig. 1. Esquema circular de la actuación ética.

Fuente: elaboración propia.

\section{Objetivos y metodología.}

Los objetivos que se quisieron cumplir son los propios del tema que se trataba, y que fueron cuatro:

- $\quad$ 01: Analizar el papel social que representa la empresa en su entorno, como motor de un desarrollo local sostenible, desde el punto de vista económico.

- O O2: Conocer qué es la ética empresarial y la necesidad de una cultura empresarial basada en ella.

- O3: Impulsar la responsabilidad individual de los directivos y potenciar un modelo de liderazgo enfocado hacia las personas, a través de la colaboración interna y externa. En este caso, tomando decisiones que afectaban a las personas, su privacidad, y el medio ambiente.

- O4: Relacionar esas decisiones con las oportunidades que brindan las tecnologías de la información para cumplir con los Sustainable Development Goals (SDGs), objetivos de desarrollo sostenible. Estos objetivos son promovidos por las Naciones Unidas, tal como muestra la International Telecommunication Union (ITU, 2017).

Para cumplir cada uno de estos objetivos, a través de la metodología LSP se fueron proponiendo varios "retos":

O1: representar una empresa que actúe de manera ética.

(cc) B EY-NC-ND 2018, Universitat Politècnica de València 
O2: a partir de los conocimientos teóricos que se explican en el aula, representar la empresa tomando el punto de vista del "bien común".

O3: tener en cuenta los grupos de interés que pueden estar afectados por la actividad empresarial que se quiere desarrollar.

O4: detectar qué SDGs se cubren con la propuesta final.

Para alcanzar los objetivos que nos proponemos, hemos creído que la mejor manera es aplicar el Aprendizaje experiencial (Kolb, 2014). Para ello, de acuerdo con De Miguel et al. (2015), la herramienta LEGO Serious Play (LSP) logra que los alumnos, a través del proceso de la herramienta, participen al 100 \%. Además, fomenta la creatividad del grupo y consigue una mayor eficiencia en los objetivos de aprendizaje y en la gestión de la comunicación grupal. Además, otros autores como Mabogunje et al. (2008) o Swann (2011), también han utilizado LSP para la toma de decisiones de una manera más participativa.

La metodología LSP parte de la creación de grupos de trabajo de no más de 6 personas. A partir de estos grupos, los retos se resuelven primero de una manera individual, y después de forma colectiva, teniendo que llegar a un acuerdo sobre el modelo final propuesto.

\section{Desarrollo de la innovación.}

En primer lugar, se les dio a los alumnos una breve introducción sobre los conceptos que se iban a tratar, diferenciando la responsabilidad colectiva (responsabilidad social corporativa o corporate social responsibility-CSR) de la responsabilidad individual (profesional).

Siguiendo la clasificación de Garriga y Melé (2004), se exponen las teorías sobre CSR en cuatro grupos:

A) Teorías instrumentales: las que se centran en conseguir objetivos económicos a través de actividades sociales. En estas teorías podríamos encontrar, por ejemplo, negocios recientes que se han basado en dar servicios o beneficiar a personas de bajo nivel económico ("The Fortune at the Bottom of the Pyramid”-BoP).

B) Teorías políticas: las que buscan un uso responsable del poder empresarial en el plano político-social. Estas teorías podrían incluir tanto las posiciones extremas de los movimientos anti-globalización, como aquellas promovidas desde organismos internacionales para concienciar a las empresas y lograr un compromiso social.

C) Las teorías integradoras: lo que pretenden es que se cubran las demandas sociales que en cada momento/país/comunidad se demanden. Su visión se centra en lo local o aquello que le afecte, teniendo en mente obtener la mejor ventaja competitiva.

D) Las teorías éticas: se centran en que solo la búsqueda del bien común es la manera de alcanzar una buena sociedad. Y para lograr el bien común hay que pensar en todos los grupos de interés (stakeholders) que se puedan ver afectados por la actividad empresarial. En su revisión del estado del arte sobre la “teoría de los stakeholders”, Freeman et al (2010)

(cc) EY-NC-ND 2018, Universitat Politècnica de València

Congreso IN-RED (2018) 
conciben ésta como una teoría aplicada a la práctica de la empresa y a la toma de decisiones de sus directivos, donde cada situación debe analizarse. Aboga por poner a la ética en el centro de la empresa y a la empresa en el centro de la ética, siendo una teoría pragmática donde no se contemplan ética y empresa como elementos aislados sino interdependientes. Dentro de estas teorías éticas, también se encontraría la de Desarrollo Sostenible propuesta por Bruntland (1987), para quien es necesario no olvidar el desarrollo de las generaciones futuras.

En el caso de los negocios internacionales, no todos los países tienen el mismo concepto de cómo hacer negocios. Entonces, ¿cómo se puede enfrentar una empresa o directivo ante un dilema ético en otro país? De acuerdo con el relativismo, nos adaptaríamos a la cultura del país en cuestión (si el país es corrupto, solo cabe ceder a la corrupción). Por otro lado, según el imperialismo sólo la forma de pensar propia (normalmente occidental) es válida y no hay que tener en cuenta la cultura de dicho país. Sin embargo, si pensamos en el respeto a los derechos humanos, una cultura intermedia nos llevaría a intentar entender la cultura de ese país pero sin dejarnos llevar a posturas que dañen más que mejoren la situación y/o que ataquen los bienes éticos universales.

De este modo, con el uso de la herramienta LSP, se les propone trabajar en grupo para resolver este reto:

En la actualidad, el "Internet de las cosas" (The Internet of Things-IoT) permite que determinados aparatos, en base a una extensa red de datos personales, presten servicios sin ayuda humana. El desarrollo de este modelo de negocio también ha llegado a los países en vías de desarrollo (BoP), lo que puede plantear oportunidades y amenazas para sus ciudadanos.

En base a algunos ejemplos subidos al espacio PoliformaT de la asignatura, se les plantea a los alumnos que, pensando en esas oportunidades y amenazas, pero sin repetir los ejemplos proporcionados, desarrollen un servicio de IoT, destinado a una comunidad que entre en el concepto de BoP, teniendo en cuenta varios aspectos:

- Que sea un servicio que pueda contribuir al desarrollo de la localidad.

- Que no suponga una invasión de la privacidad de los usuarios.

- Que sea medioambientalmente sostenible.

- Que pueda relacionarse con uno o varios de los SDGs.

En este caso, los grupos que se formaron fueron cuatro, y estuvieron compuestos por 4-6 alumnos, siendo un total de 20 alumnos. Al inicio de la actividad los alumnos están un poco desorientados porque, además de la dificultad del reto, el expresarlo con las piezas LEGO no es fácil para ellos (Imagen 1). Sin embargo, ese ejercicio intelectual les permite pensar bien todos los factores a tener en cuenta. 


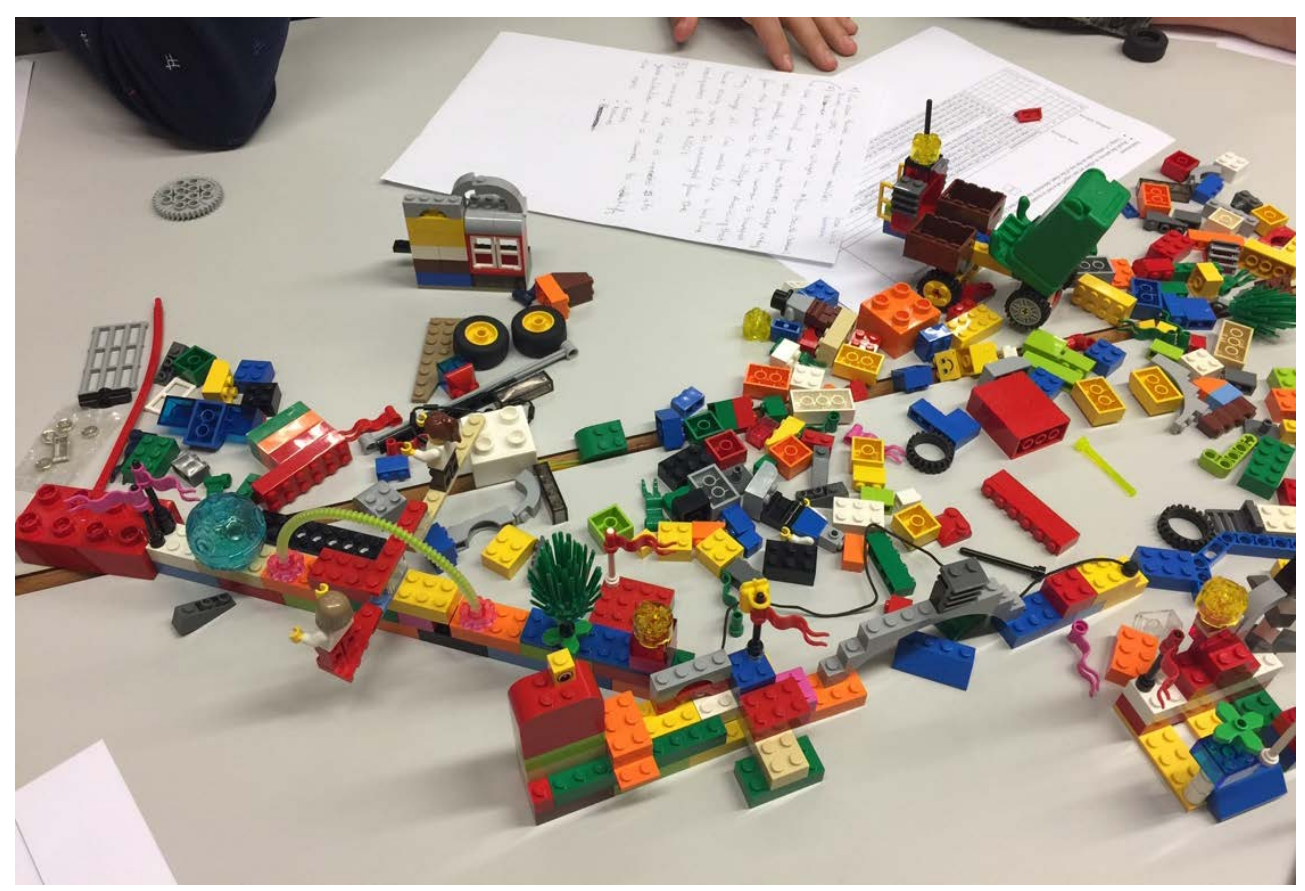

Imagen 1. Inicio de la actividad en grupo con las piezas LEGO.

Fuente: elaboración propia, curso 2017-2018.

Conforme va pasando la actividad, y con la ayuda del profesor (facilitador), el modelo va tomando forma y van integrando tanto los aspectos sociales como medioambientales, de manera que el resultado final es más rico de lo que podría pensarse en un inicio (Imagen 2).

\section{Resultados.}

El reto se desarrolla en 6 apartados:

1) Cuál es el producto o servicio que se va a ofrecer. Con qué tipo de recursos y/o energía va a funcionar. Tiene que enfocarse en un país o localidad con problemas de desarrollo. Debe ser un servicio basado en el IoT.

2) A qué personas irá destinado. Se debe de pensar en el concepto de BoP.

3) Qué tipo de datos personales son necesarios y cómo se va a manejar la información de los usuarios. Se debe de tener en cuenta el derecho a la privacidad, independientemente del país en el que nos encontremos.

4) Qué problemas éticos se pueden plantear.

5) De qué manera podrán resolverse.

6) Qué SDGs podrán cubrirse con este servicio. 


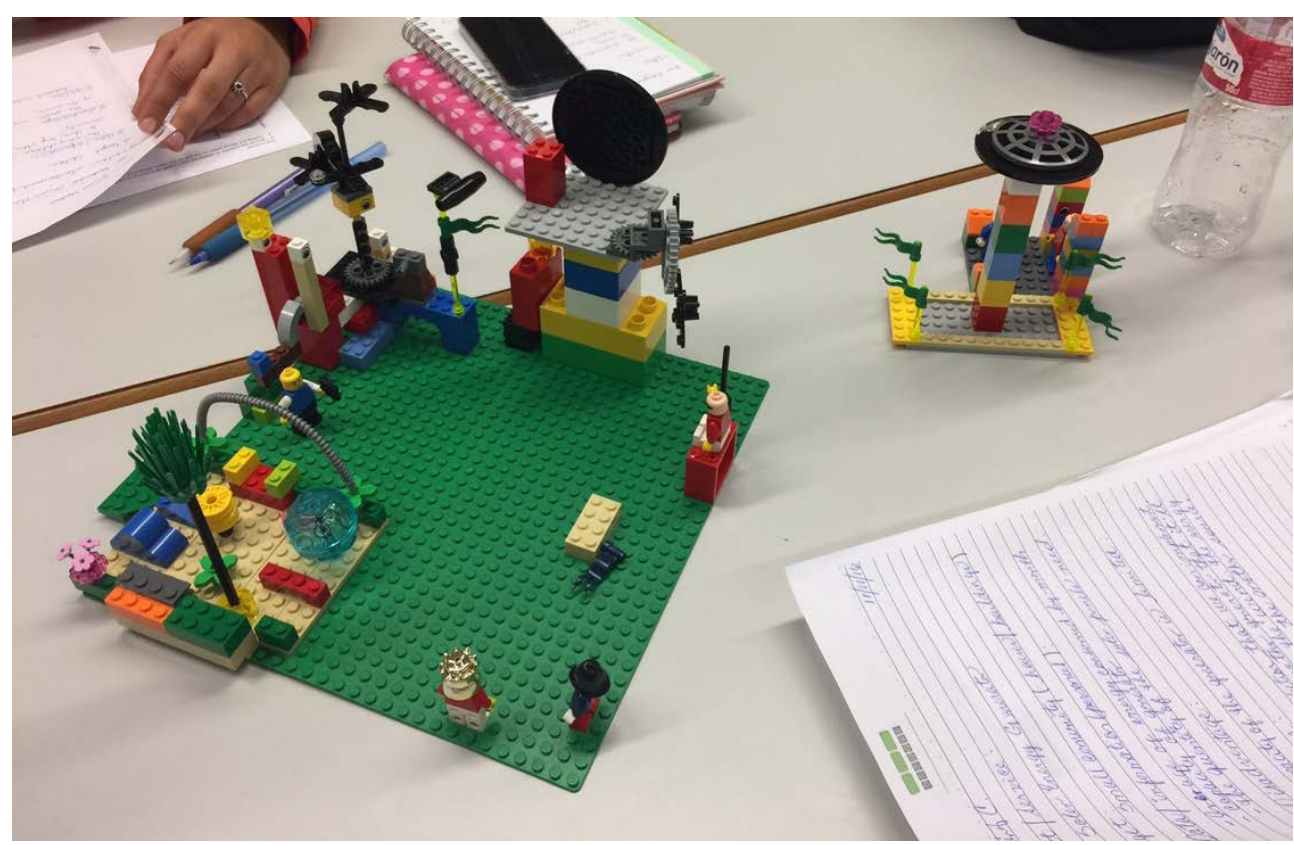

Imagen 2. Modelo propuesto por un equipo de alumnos.

Fuente: elaboración propia, curso 2017-2018.

Siguiendo el método LSP (De Miguel et al., 205), cada alumno piensa en un modelo pero después, en grupos de entre 4-6 alumnos, deben proponer un modelo común. Estos modelos se van presentando después entre los distintos grupos para lograr el feedback necesario para revisar el modelo.

Al final de esta actividad, se les pide un informe en el que expliquen los pasos que han seguido y que reflexionen sobre su decisión final (que puede ser justificada de manera distinta por cada grupo). De esta manera, podemos evaluar la competencia transversal "responsabilidad ética, medioambiental y profesional".

Como ejemplo, uno de los equipos propuso un servicio de reciclado en el que los ciudadanos pueden llevar materiales para ser reutilizados para la construcción. Estos materiales se emplearían para construir viviendas destinadas a familias pobres con menores a su cargo. Se podría hacer una aplicación (app) sencilla para saber qué materiales son necesarios, dónde dejarlos, cuándo van a estar las viviendas disponibles, cómo se va desarrollando la lista de espera, etc. De esta manera, las propias familias que reciclan se pueden ver beneficiadas. En este caso, se van a recopilar datos personales de los ciudadanos (nivel económico, menores a su cargo, domicilio, ubicación, etc), por lo que la protección de su privacidad es esencial. Esta propuesta se relacionaría, además, con los siguientes SGDs: No poverty-Industry Innovation and Infrastructure-Economic Growth-Climate. 


\section{Conclusiones.}

Si bien evaluar esta competencia siempre va a tener limitaciones, el ofrecer una práctica real en la que la toma de decisiones se base en la experiencia, tanto individual como grupal, puede ayudarnos a observar cómo los alumnos han interiorizado los conceptos explicados al inicio de la actividad.

De este modo, podemos decir que los objetivos planteados se han cubierto. En concreto:

- $\quad$ O1: se ha visto cómo la empresa puede ser motor de desarrollo incluso en entornos desfavorecidos.

- $\quad$ O2: se ha interiorizado cómo la ética empresarial es posible si la Alta Dirección lo promueve.

- O3: para el éxito de cualquier empresa, la colaboración interna y externa es primordial, y hay que tener en cuenta que las decisiones de la empresa afectan a las personas y el medio ambiente y, en este caso, además, a la privacidad de los usuarios.

- O4: relacionar esas decisiones con los Sustainable Development Goals (SDGs), nos ayuda a pensar de una manera global mientras se actúa en lo local.

Los modelos elaborados suelen ser muy ricos, en el sentido que la suma de la experiencia del grupo hace que se tengan en cuenta más factores y se presenten soluciones más creativas.

El resultado son modelos reales, que perfectamente podrían desarrollarse en países o localidad en desarrollo, de manera medioambientalmente sostenible y teniendo en cuenta la privacidad de sus usuarios. La evaluación se realiza teniendo en cuenta de qué manera han interiorizado todos los pasos y han aplicado los conceptos explicados al inicio de la sesión.

\section{Agradecimientos.}

Los autores desean agradecer a la UPV su apoyo a través del PIME 2018 “Adaptación y desarrollo de aprendizajes experienciales al contexto de las asignaturas”, dirigido por el profesor Ángel Peiró.

\section{Referencias}

DE-MIGUEL-MOLINA, M.; ALBORS-GARRIGÓS, J. CERVELLÓ-ROYO, R.; DE-MIGUELMOLINA, B.; SEGARRA-ONA, M.V. y PEIRÓ-SIGNES, A. (2015). Competencias transversales y Lego Serious Play: la necesidad de un enfoque adecuado. In-Red 2015 - Congreso de Innovación Educativa y Docencia en Red. Universitat Politècnica de València. $<$ http://dx.doi.org/10.4995/INRED2015.2015> [Consulta: 29 de marzo de 2017]

EUROPEAN UNION. Tuning Educational Structures in Europe (competences). $<$ http://www.unideusto.org/tuningeu/competences.html> [Consulta: 23 de marzo de 2017]

FREEMAN, R.E., HARRISON, J.S., WICKS, A.C., PARMAR, B.L. y DE COLLE, S. (2010). Stakeholder theory: The state of the art. Cambridge University Press.

(cc) EY-NC-ND 2018, Universitat Politècnica de València

Congreso IN-RED (2018) 
GARRIGA, E. Y MELÉ, D. (2004). Corporate Social Responsibility Theories: Mapping the Territory, Journal of Business Ethics, 53, pp. 51-71.

ITU. ICTs for a Sustainable World \#ICT4SDG. <http://www.itu.int/en/sustainableworld/Pages/default.aspx> [Consulta: 20 de noviembre de 2017]

KOLB, D.A. (2014). Experiential learning: Experience as the source of learning and development. FT press.

MABOGUNJE, A., HANSEN, P.K., ERIS, O. y LEIFER, L. (2008). Product Design and Intentional Emergence facilitated by Serious Play. DS 50: Proceedings of NordDesign 2008 Conference.

SWANN, D. (2011). NHS at Home: Using Lego Serious Play to Capture Service Narratives and Envision Future Healthcare Products. INCLUDE 2011 Proceedings.

UNIVERSITAT POLITÈCNICA DE VALÈNCIA. Competencias Transversales, competencia responsabilidad ética, medioambiental y profesional. $<$ http://www.upv.es/contenidos/COMPTRAN/info/954882normalc.html> [Consulta: 29 de marzo de 2017] 